\title{
FASTER PLASTICIZERS PRODUCTION BY MICROWAVE IRRADIATION
}

\section{A. Jermolovicius ${ }^{1}$, E. V. S. Pouzada ${ }^{1}$, E. R. de Castro ${ }^{1}$, R. B. do Nascimento ${ }^{1}$, J. T. Senise ${ }^{1}$ \\ ${ }^{1}$ Instituto Mauá de Tecnologia, Praça Mauá, 1, São Caetano do Sul, SP 09580-900, Brazil. jermolovicius@maua.br}

Keywords: microwave assisted chemical process, plasticizers, effect of microwaves on chemical kinetics.

\begin{abstract}
In this work the microwave effect of enhancing chemical reactions was applied to maleic anhydride esterification with 2-ethylhexanol-1 catalyzed by p-toluene sulfonic acid. The analysis of this reaction was simplified considering the industrial practice of withdrawn the reaction water, which makes the whole reaction act as a pseudo irreversible one. A statistical design of experiments was applied to determine an empirical chemical kinetics equation with three levels of catalyst concentration and two levels related to the kind of heating (microwave and conventional); six chemical kinetics equations (three for microwave heating and three for conventional heating) were determined. A batch/plug flow simulation was done and a spreadsheet developed for the reactor's behavior. The most interesting result was the confirmation of a synergic effect between microwaves and acid catalysis that accelerated this esterification reaction, transforming it in a fast reaction.
\end{abstract}

\section{Introduction}

Chemical industry may be conceived as a reactor surrounded by various process equipment as pumps, evaporators, dryers, distillers, crystallizers etc. All of them are necessary to transform raw materials into products. The reactor is the equipment where raw materials molecular structures are changed through a chemical reaction and the remainders act to purify the product produced inside the reactor.

Chemical kinetics data are fundamental for a good reactor project [1] because they provide the basic information to design a suitable reactor volume that fits the chemical reaction necessity of processing time, temperature, products distribution etc. Unfortunately, this kind of data are rare [2] and even rarer are data for chemical kinetics enhanced by microwaves.

Nowadays, it is well known the microwave effect that accelerates chemical reactions [3-5], but data for the complete chemical equation (equation with reaction order for all reactants, activation energy, pre exponential Arrhenius factor) are still very rare [2].

This paper was written with the aim to disclose how chemical kinetics equation may be determined and applied to the project of microwave irradiated reactors and/or conventional reactor.

\section{Plasticizers}

Plasticizers are esters of organic acids or acid anhydrides, which are used as additives in the plastics, concrete and ceramics industries to increase plasticity and or decrease viscosity of a material. Several products may act as a plasticizer, but only approximately fifty are commercial products and most of them are for PVC applications [6].

Di-2-ethylhexil maleate (DEM) was selected as an example for this study. It is produced by reaction of maleic anhydride (MA) with 2-ethylhexanol-1 (EH), usually catalyzed by acids, as p-toluene sulfonic acid (PTSA) [7]. There is also a second two step esterification reaction: firstly, MA reacts with EH producing mono-2-ethylhexyl maleate (MEM) and then MEM reacts with more EH to produce DEM. These equations are shown in (1) and (2), respectively. In industry is usual to work with a global reaction, that is the sum of these two partial reactions, as shown in (3). 
Reaction 1 is fast and irreversible, because it deals with anhydride and alcohol, producing only one product, the MEM. The second reaction is reversible because it is a reaction between a carboxylic acid and an alcohol, producing an ester (the DEM) and water, which may establish a dynamic equilibrium with their raw materials.

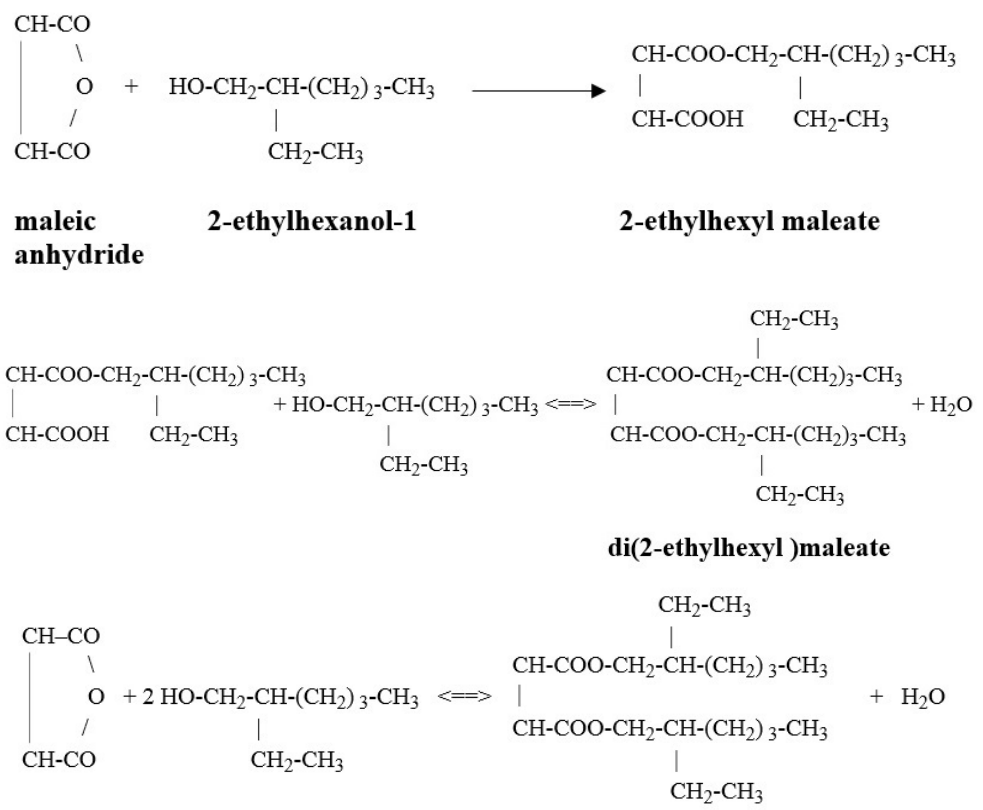

The reversibility of the second reaction is a technical problem in industry, because it reduces the total conversion of reactants. Increasing conversion of MA is done by adding xylene to the load, as a solvent, and distilling an azeotropic mixture of xylene and reaction water [7]. Elimination of this reaction water, shift the reaction equilibrium to products formation, becoming a pseudo-irreversible reaction, as shown in (4), and increasing yield in DEM.

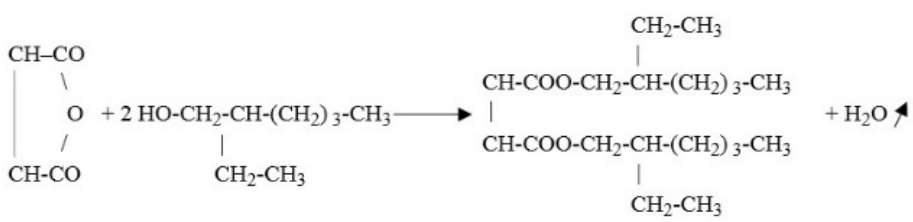

Equation (4) is the representation used in industry for controlling production of DEM and it was adopted for this study.

\section{Reaction velocity}

Chemical reaction engineering deals with reaction velocities to select and design chemical reactors for a desired capacity of a product production [1]. All project strategies are based on the chemical reaction behavior, which is well expressed by chemical kinetics equations. Supposing a non-elementary reaction (5),

$$
\mathrm{A}+\mathrm{B} \rightarrow \mathrm{R}
$$

then its reaction velocity may be expressed by (6) [1].

$$
-r_{A}=k \cdot C_{A}^{n a} \cdot C_{B}^{n b}
$$


where, $-r_{A}$ is the reaction velocity, $k$ is the velocity constant, $C_{A}$ and $C_{B}$ are the concentrations of the reactants $\mathrm{A}$ and $\mathrm{B}$, and $\mathrm{n}_{\mathrm{a}}$ and $\mathrm{n}_{\mathrm{b}}$ are the individual reaction order, related to $\mathrm{A}$ and $\mathrm{B}$. The $k$ factor is a pseudo constant, because it depends on the reaction temperature according to Arrhenius law [8]:

$$
k=k_{0} \cdot \exp (-E / R T)
$$

where, $k_{0}$ is the Arrhenius pre-exponential factor, $E$ is the activation energy, $R$ is the universal gas constant and $T$ is the temperature. Other factors can also influence this value, for example: use of catalyst and its concentration, reaction mechanism, mixing, irradiation [10]. Usually, kinetics equation is determined for specific conditions of temperature, catalysis and reactor operation. This restriction is to simplify the experimental determination of reaction velocities.

A simulation of reaction 5, shown in Figures 1-3, exemplify how kinetics parameters influence the reaction rate. Fig. 1 shows that, for a specific value of velocity constant, reactions with lower orders are faster. Fig. 2 shows that, for a specific reaction order, concentrated reactants produce faster reaction. Fig. 3 shows that higher values of velocity constant produce faster reactions.

It is possible to observe (Fig. 2) that, sometimes, high level of reactants conversion may be reached in a few minutes and also that total conversion may happen in a long reaction time. In industrial practice, the usual reactant concentration is about 10-20 M and the economic conversion is about $92 \%$ which may be reached after several hours. For this reason, in this case, the reaction is classified, in industry, as a slow reaction although its high conversions during the initial operation time, even if catalyzed.

High reactant concentrations may reduce the reaction time to reach the $92 \%$ conversion level, but it is not economical for industries. Then the option is to change the reaction conditions, as temperature, mixing, contact time. Obviously, the best procedure is to enhance the reaction rate by changing the reaction mechanism, say, changing the values of activation energy, reaction order of reactants, pre-exponential Arrhenius constant. This option means that alterations in the reaction mechanism and catalysis are the first options.

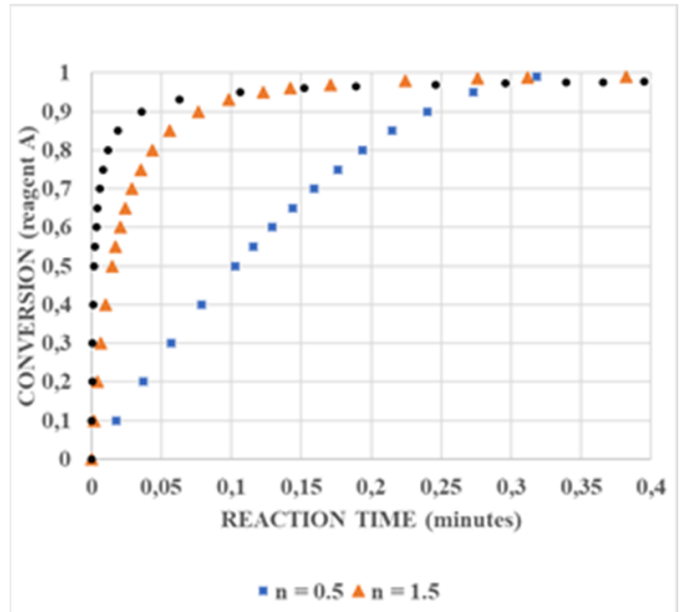

Fig.1. Reaction order $(0.5,1.5$ and 2.5$)$ effect on conversion $\left(k=17.9 ; \mathrm{C}_{\mathrm{A}}=\mathrm{C}_{\mathrm{B}}=10 \mathrm{M}\right)$. 
Nowadays, there is a new option: the microwave enhanced chemistry. There are several reports and review papers on reaction acceleration by microwaves [3-5]. Most of the reported experiments were done in small scale; unfortunately, this kind of data are not suitable for industry reaction conditions (especially in petrochemical industry). The knowledge of chemical kinetics equation of (conventional or microwave enhanced) chemical reactions can help the scale up process and the design of microwave irradiated chemical reactors.

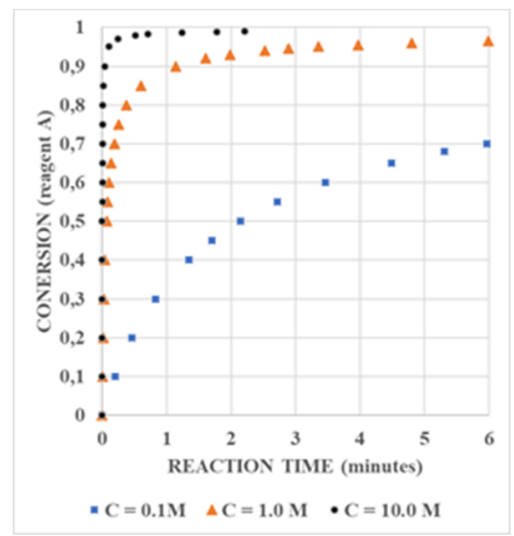

Fig.2. Reactants concentration $(0.1,1.0$ and $10.0 \mathrm{M})$ effect on conversion (reaction order $2.5, k=17.9$ ).

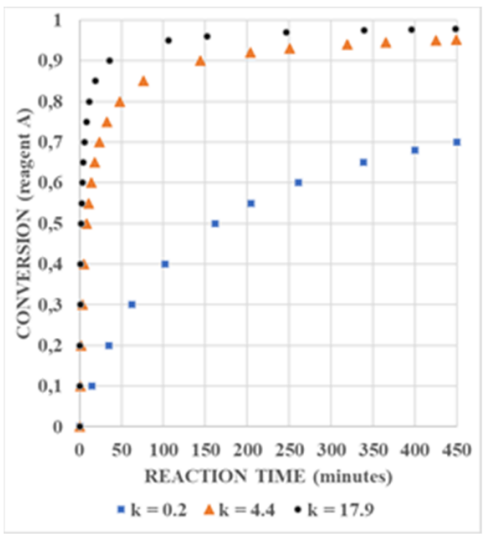

Fig.3. Velocity constant $(0.2,4.4$ and 17.9$)$ effect on conversion (reaction order $2.5, \mathrm{C}_{\mathrm{A}}=\mathrm{C}_{\mathrm{B}}=0.1 \mathrm{M}$ ).

\section{Experimental}

\section{Reactor and operational procedure in chemical kinetics experiments}

A procedure for the determination of chemical kinetics was developed in an early work [10] and is described in this paper. The reactions were performed in a well-stirred batch reactor of $500 \mathrm{~mL}$ boron silicate glass kettle vessel with internal baffles used to prevent whirlpool formation, with a top cover with four holes 24/40. A shaft of boron silicate glass rod and a 
paddle of Teflon were used. The stirrer's explosion proof electrical motor was assembled over the top of the reactor. A total reflux Allinh condenser (boron silicate glass, $60 \mathrm{~cm}$ ) with a Dean Stark flask $(25 \mathrm{~mL})$ was assembled at the vessel top. The condenser was cooled with water at room temperature. The sample collector was a $2 \mathrm{~mm}$ inner diameter boron silicate glass tube immersed into the reagents and with the other end side connected to a vacuum vessel. It was fitted at the same hole used to feed the reagents. A digital fiber optic thermometer sensor, protected by a thermometric well of boron silicate glass, was used to monitor the temperature of reactor load. The whole assembly was enclosed in an aluminum wall multimode cavity with a mode stirrer, connected to a microwave variable-power generator of $2.45 \mathrm{GHz}$. A directional coupler and a power meter measured the microwave transmitted and reflected power. During the experiments with conventional electric heating, the reactor was wrapped with an electric tape with temperature control.

The experiments were performed with loads of $300 \mathrm{~mL}$ of solution of MA and PTSA in xylene, which were heated up to the reaction temperature. Simultaneously the right quantity of EH was heated to 139 or $143{ }^{\circ} \mathrm{C}$ (according to the test planned, as shown in Table 1) in an electrical heated separatory funnel. When the temperature in the reactor and in the separatory funnel reached 139 or $143{ }^{\circ} \mathrm{C}$ the $\mathrm{EH}$ was introduced into the reactor over the MA solution and the time count started. This pre-heating of raw materials was done because it was observed in a preliminary experiment that 12 minutes were necessary to heat the load from room temperature up to $143^{\circ} \mathrm{C}$.

The azeotrope vapor of xylene and water was condensed in the Allinh condenser and the condensed azeotrope broken in two phases: an upper one with xylene and a lower with water. These phases were decanted in the Dean Stark flask and the condensed xylene was returned to the reactor and the water drawn and discarded. At the beginning of each esterification, the Dean Stark was filled up with dry xylene. During the esterification, the Dean Stark flask water level was maintained at a maximum level of $2 \mathrm{~mL}$ by intermittently drawing off the water.

The samples were taken in a 1 minute time step, during the total processing time of 10 minutes; they were analyzed with a near infrared (NIR) spectrometer calibrated for maleic anhydride.

The concentration of reactants solutions and the reaction temperature of each test are described in the next section. The solvent used was dry xylol. The experiments with conventional electric heating and with microwave irradiation were done at the same temperatures. The effective microwave power was $1.33 \mathrm{~W} / \mathrm{g}$ for $139{ }^{\circ} \mathrm{C}$ and $1.69 \mathrm{~W} / \mathrm{g}$ for $143^{\circ} \mathrm{C}$.

\section{Experimental determination of complete chemical kinetics equation}

Simplifying the kinetics analysis, the esterification of MA by EH, catalysed by PTSA, with withdrawn of reaction water, was assumed as a pseudo irreversible reaction. This simplification is expressed in (4).

Assuming that (a) the concentration of PTSA is constant, (b) the reaction is non-elementary and (3) that it is processed isothermally, then it was possible to write a chemical kinetics equation with three variables (1/T, CMA an CEH) as in (8),

$$
-r_{M A}=k_{0} \cdot \exp (-E / R T) \cdot C_{M A}^{n a} \cdot C_{E H}^{n b}
$$

where $\mathrm{r}_{\mathrm{MA}}$ is the rate of consumption of MA, $\mathrm{k}_{\mathrm{o}}$ is the Arrhenius pre exponential factor, $\mathrm{E}$ is the activation energy $(\mathrm{kcal} / \mathrm{mol}), \mathrm{T}$ is the reaction temperature $(\mathrm{K}), \mathrm{C}_{\mathrm{MA}}$ and $\mathrm{C}_{\mathrm{EH}}$ are molar concentrations of MA and HE respectively, $n_{a}$ and $n_{b}$ are the reaction orders for MA and HE respectively. Then, equation 8 was linearized by logarithm application and resulted in equation 9, which is a multilinear equation and may be obtained by regression analysis. 


$$
\log \left(-r_{M A}\right)=\log \left(k_{0}\right)-(E / R T) \cdot \log (e)+n_{a} \log \left(C_{M A}\right)+n_{b} \log \left(C_{E H}\right)
$$

Initial velocity method [1] and multilinear regression based on $2^{3}$ complete factorial design [11] were applied together to establish an experimental chemical kinetic equation; care was take to use logarithms as regression parameters of the initial reaction rate, concentration variables and the inverse of temperature in Kelvin. Table 1 shows the experimental design for determination of one chemical kinetics equation. The laboratory procedure for this experiment was described above.

Table 1. Experimental conditions for the factorial design.

\begin{tabular}{ccccccc}
\hline $\mathrm{a}$ & $\mathrm{b}$ & $\mathrm{c}$ & $\mathbf{C}_{\mathbf{M A}}(\mathbf{M})$ & $\mathbf{C}_{\mathbf{E H}}(\mathbf{M})$ & $\mathbf{T}\left({ }^{\circ} \mathbf{C}\right)$ & $\mathbf{1} / \mathbf{T}(\mathbf{1} / \mathbf{K})$ \\
\hline+ & - & - & 2 & 0.5 & 139.2 & 0.002426 \\
- & - & - & 1 & 0.5 & 139.2 & 0.002426 \\
+ & - & + & 2 & 0.5 & 142.8 & 0.002404 \\
- & - & + & 1 & 0.5 & 142.8 & 0.002404 \\
+ & + & - & 2 & 1 & 139.2 & 0.002426 \\
- & + & - & 1 & 1 & 139.2 & 0.002426 \\
+ & + & + & 2 & 1 & 142.8 & 0.002404 \\
- & + & + & 1 & 1 & 142.8 & 0.002404 \\
\hline
\end{tabular}

The above described experimental procedure allows us to draw the curve of MA concentration decay, during the time of test. Each curve has ten results of MA concentration determined every minute, for ten minutes. This curve was numerically derived to obtain instantaneous values of the reaction speed. From these data, the initial velocity (at zero time) is used to process the regression analysis.

This experimental design was repeated six times in two sets of experiments: one with conventional electric heating and another with microwave heating. Each set had three levels for PTSA concentration: $0.012 \mathrm{M}, 0.006 \mathrm{M}$ and without PTSA. Each repetition was done in duplicate. The order of execution within a repetition was random with no restrictions.

\section{Reactor simulation for DEM production}

It is difficult to visualize the global effect of chemical kinetics on a reactor design by analyzing only their kinetic parameters. The effect of these parameters on the reactor's physical dimensions is very abstract to conceive. This situation consumed long time to be solved. The solution found was to analyze the reactor behavior instead of its chemical kinetic equation parameters. Therefore, an Excel spreadsheet was developed to allow the simulation of the reactant conversion for different chemical kinetic equations and different operation conditions.

For the simulation of the present case of DEM production it was assumed that the reactor is ideal, operating in isothermal condition and with negligible change of the volume of the reactants [1]. This spreadsheet basically resolves the equations for batch or plug flow reactor. Considering all the above restrictions, then batch and plug flow reactors have the same project equations [1].

The chemical equation is introduced in the spreadsheet and the variables as reactants concentration, temperature and molar relation between reactants can be changed. As a result, it shows the reaction time necessary to obtain a specified conversion of the limiting reagent in a batch or plug flow reactor. 


\section{Results and discussion}

The initials results were the consumption curves for MA. There were six pairs of duplicates, one pair for each combination of two kinds of heating and three PTSA concentrations. These data were numerically derived to produce the MA consumption rate curves which led to the initial velocity figure, and, finally, the empirical chemical kinetics for MA esterification by EH with PTSA catalyzing and microwave/conventional electric heating, as stated by (8). Table 2 shows these data.

In the case of conventional electric heating the activation energy decreases to approximately $90 \%$ of its initial value (without PTSA) as the PTSA concentration increases. This is the expected effect; it seems that this decrease follows a parabolic curve. An unexpected result occurred when microwave heating was applied. The activation energy decreased only $25 \%$ of its initial value and has a maximum value in the middle of PTSA concentration range, when it increased approximately $59 \%$ of its initial value. This fact requires a more detailed future study.

Table 2. Chemical kinetics parameters for MA esterification with EH in (8).

\begin{tabular}{cccccc}
\hline PTSA (M) & Heating & $\mathbf{k}_{\mathbf{0}}$ & $\mathbf{E}$ (kcal/mol) & $\mathbf{n}_{\mathbf{M A}}$ & $\mathbf{n}_{\mathbf{E H}}$ \\
\hline 0 & Electrical & $2.29 \mathrm{E}+78$ & 149.31 & 0.89 & 2.47 \\
0 & MW & $6.66 \mathrm{E}+14$ & 25.65 & 1.59 & 2.24 \\
0.006 & Electrical & $1.39 \mathrm{E}+25$ & 46.30 & 0.56 & 2.18 \\
0.006 & MW & $6.90 \mathrm{E}+21$ & 40.73 & 1.04 & 1.98 \\
0.012 & Electrical & $2.96 \mathrm{E}+08$ & 15.87 & 0.25 & 0.65 \\
0.012 & MW & $2.36 \mathrm{E}+52$ & 19.31 & 0.25 & 0.05 \\
\hline
\end{tabular}

The Arrhenius' pre-exponential factor had different responses to the PTSA concentration increase. For the conventional electric heating case, this value showed a strong decrease while for microwave heating it increased and, at 0.012 M PTSA concentration, its value was near to the conventional electric heating value without PTSA.

The reaction orders decreased with the PTSA concentration increase for both kind of heating. The order with respect to MA decreases, apparently, as a linear law. The order related to EH decreased, apparently, as a second order curve. Using microwave heating, the order related to MA is higher than that of the conventional heating; the order related to $\mathrm{EH}$ is lower than that of the conventional heating. The global order, up to 0.006 M PTSA concentration, is higher than the value of the conventional electric heating, but, beyond this point, an inversion occurred and the global order using microwaves decreased approximately $67 \%$ of the conventional heating value order. In practical terms all those observations did not show clearly this effect; putting differently: the best operation condition for DEM production was not determined.

The conversion of MA was simulated for all six options (three PTSA concentrations and two kinds of heating) using the developed spreadsheet. It was assumed an isothermal operation at $140{ }^{\circ} \mathrm{C}$ with a load of MA $10 \mathrm{M}$, EH $20 \mathrm{M}$, PTSA 0.006 (or $0.012 \mathrm{M}$ ) and dry xylene as solvent. The results are shown in Fig. 4, including a window with time scale magnified to show the first minute of the reaction. 


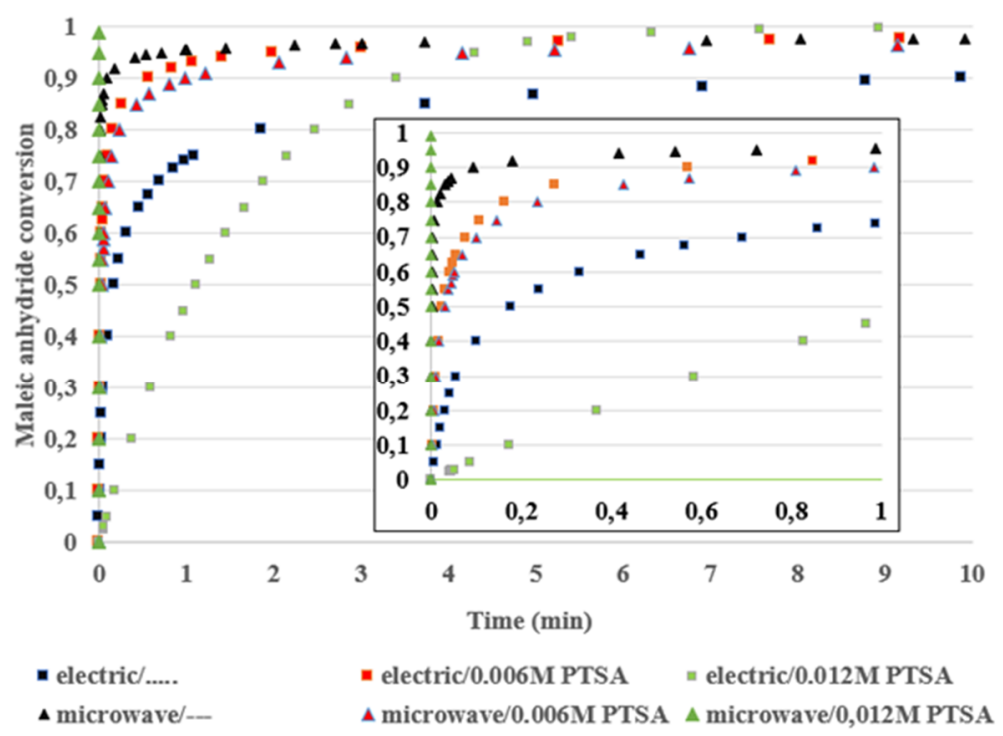

Fig. 4. Simulation of MA conversion I esterification with EH, catalyzed by PTSA and at $140{ }^{\circ} \mathrm{C}$, based on empirical chemical kinetic equations.

Curves of the electric heating show that, without PTSA, the reaction is a slow one for the industry point of view, because of the $80 \%$ plateau. Introducing PTSA at $0.006 \mathrm{M}$, it becomes a little bit faster but not enough to change the slow attribute. With PTSA at $0.012 \mathrm{M}$, it shows an initial 3-min time interval with slower figures compared to the uncatalyzed reaction, and afterwards it went to a complete conversion in nine minutes.

Introducing the microwave heating, without PTSA, the reaction becomes faster than the conventional heated reaction without PTSA. This shows a catalytic effect of microwaves alone on this esterification, being stronger than the effect of PTSA at $0.006 \mathrm{M}$. After three minutes, the microwave enhanced reaction and the conventional heated reaction, both catalyzed with PTSA $0.006 \mathrm{M}$, show the same reaction speed and both may be considered slow reactions.

No enhancement was observed with PTSA at $0.006 \mathrm{M}$ when microwave was applied as shown by the overlapping of the three curves: electric and microwave heating with PTSA at $0.006 \mathrm{M}$ and microwaves heating without PTSA. In fact, the addition of PTSA at $0.006 \mathrm{M}$ under microwave radiation had a bad result compared to the pure microwave application without catalyst. This phenomenon may be related to the unusual behavior of the activation energy observed in the reaction with microwaves and PTSA at $0.006 \mathrm{M}$ in Table 2 and analyzed above. Consequently, a plain microwave application is better than adding catalyst PTSA.

A complete conversion was reached at $5 \times 10^{-42}$ minutes by applying microwaves with PTSA at $0.0012 \mathrm{M}$. This curve is overlapping the vertical axis in Fig. 4. This is a real fast reaction and may be understood as a flash reaction. This phenomenon may be related to a synergic effect between microwaves and PTSA that reduces global reaction order and increase Arrhenius' pre exponential factor, as observed in Table 2.

\section{Conclusion}

Microwaves enhanced the esterification of MA with EH, either with or without catalysis of PTSA, as shown in Fig. 4. An important result is that a probable synergic effect between microwaves and PTSA can transform this reaction into a fast one, also shown in Fig. 4. 
Another interesting phenomenon was observed: the reaction under microwave irradiation and PTSA at $0.006 \mathrm{M}$ had the same velocity as the uncatalyzed enhanced esterification and microwave irradiated with PTSA at $0.006 \mathrm{M}$. So, it is better to apply plain radiation than use PTSA at $0.006 \mathrm{M}$. This fact may be explained by the odd behavior of the activation energy in the interaction of microwaves and PTSA at $0.006 \mathrm{M}$, as shown in Table 2. Careful observation of Table 2 show that microwaves can change the mechanism of this esterification because all parameters vary their values at different exposition to microwave energy.

\section{Acknowledgements}

The authors would like to thank C. Tognela. Support from Instituto Mauá de Tecnologia - IMT - and from Fundação de Amparo à Pesquisa do Estado de São Paulo FAPESP - (Grant 2017/24238-7) are gratefully acknowledged.

\section{References}

1. Levenspiel, O. Chemical Engineering Reaction. 3ed, USA, John Wiley, 1999.

2. Elsevier, Science Direct. www.sciencedirect.com, (accessed in 7 April, 2019).

3. Senise, J.T., Jermolovicius, L. A. J. Microw. Optoelectron. 2004, 3, 97-112.

4. Stefanidis, G. D., Muñoz, A. N., Sturm, G. S. J., Stankiewicz, A. Rev. Chem. Eng. 2014, 30, 233-259.

5. Kappe.O. Chem. Rec., 2018, 18, 1-26.

6. Ash, M., Ash, I. Plasticizers, Stabilizers and Thickeners. USA, Ed. Arnold, 1989.

7. Groggins, P. H. Unit Processes in Organic Synthesis. 5ed, Tokyo, McGraw-Hill, Kogakusha, 1958.

8. Castellan, G. W. Physical Chemistry. USA, Addison-Wesley, 1970.

9. Glasstone, S. Tratado de Quimica Fisica. 6 ed, España, Aguilar, 1966.

10. Jermolovicius, L. A., Senise, J. T., Castro, E. R. Proc. AMPERE 2005.

11. Montgomery, D. C. Design and Analysis of Experiments. 6 ed, USA, John Wiley, 2005. 